Europhys. Lett., 23 (2), pp. 99-104 (1993)

\title{
Plasmon Effects in Solid-State Radiation Detectors.
}

\author{
T. C. Choy $\left({ }^{*}\right)$ and A. M. Stoneham (**) \\ (*) Department of Physics, Monash University - Claytom, Victoria 3168, Australia \\ (**) AEA Technology, Harwell Laboratory - Oxfordshire OX11 ORA, England
}

(received 14 October 1992; accepted in final form 18 May 1993)

PACS. 29.40P - Semiconductor detectors.

PACS. 29.70G - Energy loss and energy range relations.

PACS. 71.45G - Exchange, correlation, dielectric and magnetic functions, plasmons.

\begin{abstract}
We have examined the role of plasmons on the electron energy response of solid-state ( $\mathrm{Si}$ and $\mathrm{Ge}$ ) radiation detectors. We found that at the level of parts per thousand, internal-conversion electron calibration techniques do not suffice to yield an adequate response function. In particular, spectral distortions in the detection of low-energy beta-particles have been found which are not accounted for by the usual calibration methods. Thus, a small but significant error can arise from energy loss to low-energy plasmons in $\mathrm{Si}$ and Ge detectors. The proximity of the plasmon energy to the end-point singularity and the quadratic form of the beta-decay spectrum may account for the effect interpreted as a $17 \mathrm{keV}$ neutrino. Similar errors can also arise in other subtle solid-state measurements as, for example, in the X-ray edge absorption and emission spectra of metals and semiconductors.
\end{abstract}

Introduction. - The high-energy detection efficiency of semiconductor solid-state detectors like $\mathrm{Si}(\mathrm{Li}), \mathrm{Ge}(\mathrm{Li})$ and $\mathrm{HPGe}$ has made magnetic spectrometers nearly obsolete as standard radiation detectors (see, for example, Knoll [1]). As pointed out by Koonin [2], a parts-per-thousand accuracy in spectral shape and detector response is now demanded by sensitive nuclear detection experiments like beta-decay spectra [3-6] and others. Unfortunately, this accuracy has not been demonstrated sufficiently well enough to ensure an unambiguous interpretation of results, like the recent $17 \mathrm{keV}$ neutrino anomaly, which is a subject of controversy (see, for example, [7]). Despite the fact that careful-energycalibration curves are usually run as a matter of routine in nuclear measurements, we shall demonstrate that an inherent solid-state phenomenon due to low-energy plasmons may escape correction. As a consequence, the data are subject to a systematic error which manifests particularly near singularities in the spectrum of beta-decay. The role of low-energy collective excitations, like plasmons, has not been studied in detail for semiconductor detector materials (see Bichsel [8] for a review of the state of the art) to the required sensitivity level. Our argument is based on the known plasmon decay processes in a solid, which rely mainly on the electron-electron interactions. We found that there is a modest transfer of energy to plasmons (the natural modes of oscillation of the electron gas in the solid, here the oscillations of the electrons in the valence band of $\mathrm{Si}$ and $\mathrm{Ge}$ ), which can decay without generation of charged carriers. It is such carriers which separate and are 
measured in detector operation. Of course, not all plasmons are uncorrected for. In fact, most are already included in the energy calibration. Only a small fraction with long wavelengths can escape detector correction (see below).

The main thrust of our paper is this. The energy loss of fast electrons in solids contains essentially two components. One is electron-hole pairs, the other is plasmon excitation. The two components need not be independent: plasmons may decay to produce electron-hole pairs, and if this were the only decay mechanism, the route by which the carriers are produced would be of no concern to the user of the detector and also of no interest here. However, if some plasmons can lose energy in other ways which do not yield charge separation into observed charge carriers, or if the lifetime for some plasmons is much longer than a typical electron-hole energy collection time, there will be an effect on the observed spectrum. In the next section we shall argue, on the basis of known properties of plasmons, that this is in fact the case. In the third section we shall, without loss of generality, introduce a simplified model for the above plasmon energy loss as a (statistically independent) Poisson process. We emphasize that for this paper we are not so much concerned with the plasmon process in detail (hence the use of a simplified model). Rather our main interest is to demonstrate its effects on the measured spectrum, which seem to play a subtle role in the case of the beta-decay spectral shapes.

Key properties of plasmons. - We start from the key properties of plasmons and their generation by fast electrons. The plasmon energy $\varepsilon_{\mathrm{p}}$ is determined by the electron density, and has a value of 16 to $17 \mathrm{eV}$ in $\mathrm{Si}$ and $\mathrm{Ge}$, the standard detector materials. The plasmon energy is significantly larger than either the energy $I$ to create an electron-hole pair ( $3.76 \mathrm{eV}$ for $\mathrm{Si}, 2.96 \mathrm{eV}$ for $\mathrm{Ge}[9])$ or than the band gap $(1.16 \mathrm{eV}$ for $\mathrm{Si}$ and $0.74 \mathrm{eV}$ for $\mathrm{Ge}$ at $100 \mathrm{~K}[10])$. The similarity of plasmon energy in Si and Ge means that the mean free path $\Lambda(E)$ for plasmon generation by fast electrons of energy $E$ is very similar in the two materials. Typically (see below) $A$ varies from about $140 \mathrm{~nm}$ (at $100 \mathrm{keV}$ ) to $250 \mathrm{~nm}$ (at $200 \mathrm{keV}$ ). These mean free paths are in fact much less than the total particle energy range $R(E)$. We can readily compute the latter from the integral over the inverse stopping power, in accordance with the classic Bethe formula. With the above values, $R(100 \mathrm{keV})$ is about $58 \mu \mathrm{m}(\mathrm{Si})$ and $28 \mu \mathrm{m}(\mathrm{Ge}) ; R(200 \mathrm{keV})$ is about $215 \mu \mathrm{m}(\mathrm{Si})$ and $104 \mu \mathrm{m}(\mathrm{Ge})$. Such values are in good agreement with the data of Mukoyama [11]. The range $R(E)$ is therefore about three orders of magnitude larger than $\Lambda(E)$, the mean free path for plasmon generation. Substantial numbers of plasmons will be generated, though some will simply decay to yield observable carriers or lead to effects already included in detector calibration.

The main processes of energy collection in detectors come directly from the electron-hole pairs generated and indirectly from those plasmons with wave vectors larger than $q_{c}$, where $q_{\mathrm{c}}$ is the highest wave vector for which plasmons are well defined, being the value at which the plasmon dispersion curve (fig. 1) merges with the electron-hole continuum. The lifetime of the short-wavelength plasmons ( $q_{\mathrm{c}}$ is about $11 \mathrm{~nm}^{-1}$ for the systems we are considering) is about $0.1 \mathrm{fs}$, far shorter than the mean Rutherford collision time for energy loss $I$, which is around $10 \mathrm{ps}$ at $100 \mathrm{keV}$. However, the plasmon reciprocal lifetime varies as the square of the wave vector [12], so longer-wavelength plasmons are longer-lived. Hence we can see that optical wavelength plasmons can act as an energy sink, with lifetimes much longer than the passage time of the electron. The plasmons involved are therefore those in the region $\delta$, as indicated in fig. 1 , which have a maximum wave vector of $\bar{q}$. Their contribution to the total plasmon energy loss is small, of the order of parts per thousand. The smallness of the loss is why the effect is not seen in internal-conversion (IC) measurements, where losses to plasmons are masked by larger effects like electron backscattering [13]. This can be seen by an elementary argument. Suppose that the real loss is a sum of two Gaussian components, 


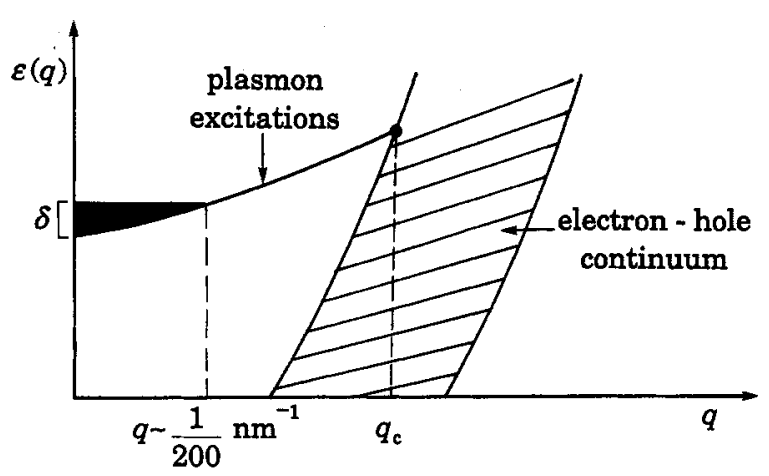

Fig. 1.

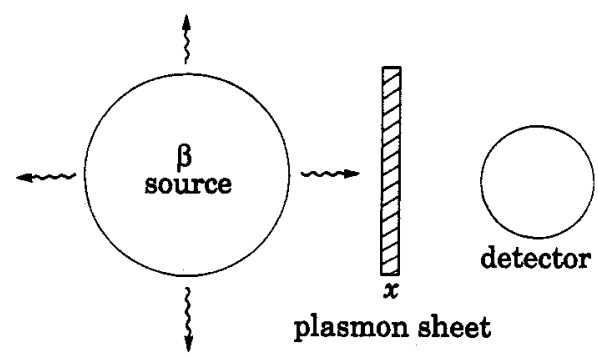

Fig. 2.

Fig. 1. - Schematic representation of the excitation energy spectra $\varepsilon(q)$ for the energy collection processes in solid-state $\mathrm{Ge}$ and $\mathrm{Si}$ detectors. The main feature is an electron-hole continuum (with a semiconductor band gap) and a plsmon $\varepsilon_{\mathrm{p}}(q)$ spectrum; these merge at wave vector $q_{\mathrm{c}}$. The dark region of for wave vectors below $\bar{q}$ represents energy loss due to long-wavelength plasmons as discussed in the second section.

Fig. 2. - Schematic representation of the model for the plasmon energy loss in region $\delta$ as shown in fig. 1. The plasmon sheet might correspond to a near-surface region of a few times $100 \mathrm{~nm}$ thickness (see the second section).

one of width $625 \mathrm{eV} \mathrm{[6]} \mathrm{due} \mathrm{to} \mathrm{electron} \mathrm{backscatter,} \mathrm{the} \mathrm{other} \mathrm{of} \mathrm{width} 32 \mathrm{eV}$ (see below) from plasmon losses. The full width from both mechanisms is the root mean square of the two components, $625.8 \mathrm{eV}$ : unless measurements are made to an accuracy of $0.5 \mathrm{eV}$ or better, our proposed plasmon effect will be undetectable in IC energy checks. By comparison with the above background, the mean plasmon loss we propose is of the order of 1 to 2 plasmon energies in the $120 \mathrm{keV}$ region and normally this would lead to insignificant errors in the experimental resolution function for most measurements. However, near to a spectral singularity, notably the maximum energy cut-off $W$ in beta-decay, we shall see that the singularity conspires with the above plasmon loss and the distortions are enhanced. Although our results above are based entirely on incident-electron-energy loss in the detector solid, the same analysis remains valid for other types of radiation detections. It should be considered, for example, in the study of the X-ray absorption edge in metals, if effects at high sensitivity are sought, as in determining power laws at the edge singularity [14].

Estimate of effects. - We now examine the effect on the spectrum due to energy loss to plasmons in the energy sink $\delta$. Our simplified model assumes a sheet of thickness $x$ of approximately $\bar{q}^{-1}$ (see fig. 1), in which the incident fast electrons are scattered (fig. 2), giving an energy loss prior to the deposition of the major part of their energy. In the last section we argued that the plasmons concerned are mainly of optical wavelengths, hence we shall adopt the value of $x$ of about $200 \mathrm{~nm}$, though it could be longer. One picture is that this region may be a near-surface layer, but, as emphasized earlier, we do not need to presume such a detailed model for the purpose of this paper.

Since the plasmon scatter processes occur prior to energy deposition, the measured energy spectrum must involve convolution with a "plasmon loss function", $f(x, \Delta)$, which is small compared to other processes, including the main bulk plasmon loss. The loss function $f(x, \Delta)$ is the probability per unit energy that a particle with initial energy $E$ will lose an energy between $\Delta$ and $\Delta+\mathrm{d} \Delta$; it is normalised so that the integral over $\Delta$ of $f(x, \Delta)$ is unity. 
Now the loss function $f(x, \Delta)$ can be related to the microscopic probability $\omega(\varepsilon, E)$ that an electron of energy $E$ loses energy $\varepsilon$ by plasmon scattering, per unit energy per unit length [15]:

$$
\omega(\varepsilon, E)=\frac{1}{\Lambda(E)} \delta\left(\varepsilon-\varepsilon_{\mathrm{p}}\right),
$$

where, for $\Lambda$ in atomic units,

$$
\Lambda^{-1}(E)=\frac{\varepsilon_{\mathrm{p}}}{4 E} \ln \left(\frac{4 E \varepsilon_{\mathrm{c}}}{\varepsilon_{\mathrm{p}}^{2}}\right) .
$$

From recent electron energy loss spectroscopy measurements, the numbers are very similar for $\mathrm{Si}$ and Ge. Here we quote the numbers of Raether [16], which give $\varepsilon_{\mathrm{p}}$ of order $17 \mathrm{eV}$, and a kinetic energy $\varepsilon_{\mathrm{c}}$ at wave vector $q_{\mathrm{c}}$ of $4.11 \mathrm{eV}$ for a $q_{\mathrm{c}}$ of $11 \mathrm{~nm}^{-1}$. Two assumptions are made here, one is that of an infinitely long-lived plasmon whose energy dispersion is therefore neglected [15] and a second where we shall assume in the interest of simplicity that the energy loss is an independent Poisson process. If (1) holds, the latter can be justified to a certain extent via Landau straggling theory [17], in view of the smallness of $x / \Lambda\left(^{1}\right)$. However, the first assumption (and thus the validity of (1) in general) is satisfactory only as a starting point, for it is not strictly in accordance with our discussions earlier in the second section concerning plasmon lifetimes. We shall not pursue these details here, as it would involve abandoning the simplified model and require sophisticated theoretical calculations for cross-sections of the kind discussed in the recent literature [19], in which the complete plasmon loss function in the detector has to be calculated, as opposed to our simplified surface sheet model. Since our emphasis is on the effects of this energy loss we shall proceed as follows. Assuming $f(x, \Delta)$ to be a discrete Poisson distribution, with contributions corresponding to the emission of different numbers of plasmons (cf. [15], fig. 4.2),

$$
f(x, \Delta)=\frac{\exp \left[-\frac{x}{\Lambda}\right]}{\varepsilon_{\mathrm{p}}} \sum_{n=0}^{\infty} \frac{1}{n !}\left(\frac{x}{\Lambda}\right)^{n} \circ\left(\frac{\Delta}{\varepsilon_{\mathrm{p}}}-n\right) .
$$

Note that, as we have fixed $x$ as $200 \mathrm{~nm}$ on the basis of optical-plasmon lifetimes, we shall have no other parameters in the theory. Using eq. (2) for $\Lambda(E)$, we can obtain the distribution $f(x, \Delta)$. We now turn to the expression for the observed spectrum $N(E)$ in terms of the initial spectrum $N_{\mathrm{s}}(E)$ and the loss function $f(x, \Delta)$ :

$$
N(E)=\int_{0}^{\infty} \mathrm{d} \Delta N_{\mathrm{s}}(E+\Delta) f(x, \Delta) .
$$

Using eq. (3) and thereby specialising to the case $x \leqslant \Lambda$, as in the case of beta-decay at the $150 \mathrm{keV}$ range, we find now that

$$
N(E)=N_{\mathrm{s}}(E) C(E),
$$

where $C(E)$ is a measure of the distortion from our uncorrected plasmon loss. For the case of

(1) The Landau loss function can be evaluated analytically in this case by contours and reduces to a Pekarian distribution in general. This integral is also a special case of one evaluated in the theory of optical lineshapes for the Huang-Rhys model of defects in solids. See [18]. 


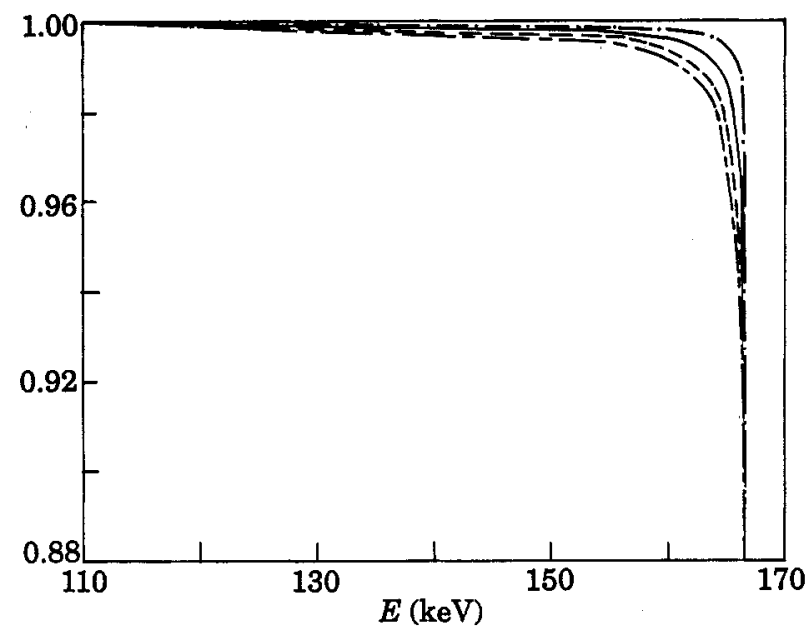

Fig. 3. - The function $C(E)$ which relates the observed beta-spectrum $N(E)$ to the actual incident spectrum $N_{\mathrm{g}}(E)$ (eq. (8)). The function saturates at the $n=4$ plasmon absorption curve for $x / \Lambda=2$ due to the large $n !$ in eq. (5). The cut-off $W$ was taken as $167 \mathrm{keV} .-\cdot-n=1,-n=2,---n=3$, $--n=4$.

beta-decay [20] for which $N_{\mathrm{s}}(E)$ has a quadratic, i.e. $(W-E)^{2}$ energy dependence, then $C(E)$ has the form

$$
C(E)=\exp \left[-\frac{x}{\Lambda}\right] \sum_{n=0}^{\frac{(W-E)}{\varepsilon_{\mathrm{p}}}}\left(1-\frac{n \varepsilon_{\mathrm{p}}}{(W-E)}\right)^{2} \frac{1}{n !}\left(\frac{x}{\Lambda}\right)^{n} .
$$

The plot of $C(E)$ is shown in fig. 3 for a fixed value of $x / \Lambda=2$, as we have found that the energy dependence of $A$ has no significant effects in this simplified model for the energy range shown in the figure. We see that there is a distortion error of about several parts per thousand near to the cut-off $W$ which was taken as $167 \mathrm{keV}[6]$. The shape function $C(E)$ in fact saturates at the 4-plasmon absorption curve due to the large factorial $n$ in eq. (6). Although the model appears simplified, we can however conclude that plasmon loss corrections are crucial for detailed studies of end-point or edge spectrum behaviour using solid-state detectors.

Conclusion. - We stress that the plasmon effect we propose is very small though potentially important. It appears that experimental efforts have not adequately addressed this issue regarding the detector response and other factors [2]. This is not surprising since our energy loss is only of one to two plasmons (about $32 \mathrm{eV}$ ), which manifests itself in the linewidth increase of only $0.8 \mathrm{eV}$ (see the second section). In the case of beta-decay and the possible existence of a $17 \mathrm{keV}$ neutrino, this level of energy calibration accuracy is also demanded over a rather wide energy range of about $17 \mathrm{keV}$, which makes stringent demands on the stability of the instruments. It does not appear that this can be achieved using IC calibration lines. Unfortunately, as we have shown, this minute correction to the experimental response function not obtainable via the IC energy calibration procedure can result in distortions near to the beta-spectrum cut-off $W$. If uncorrected for distortion, the observed form of the beta-decay spectrum might mistakenly be interpreted as giving evidence for a finite neutrino mass. Similar errors could also arise in the study of X-ray edge 
absorption spectra. Qualitatively, we see that the analysis can be carried over by changing the quadratic term in eq. (6) to one with an exponent $\alpha \sim 0.25$ [14]. However, in this case both the exponent and the X-ray plasmon cross-section are smaller. Hence the spectral distortions we have described are likely to be less severe than in the case of beta-decay. Even though our analysis in the previous section employs a simplified model, clearly a more sophisticated calculation, in which the plasmon lifetime differences would emerge from $a b$ initio band structures [19], will not affect our main results. Thus we conclude that we have identified an anomaly due to plasmon loss in the detector which conspires with the end-point singularity that is likely to be significant for detailed studies of beta-decay spectra. Indeed, the anomaly may partially or totally explain the recent controversy over the existence of a $17 \mathrm{keV}$ neutrino [7]. Our results suggest that energy checks for detector response must be made with much higher resolution, and independent experiments are needed to verify small energy losses from collective excitations.

TCC would like to thank Prof. B. Bolton in Monash University, Dr. N. A. JELLEY and Prof. Sir R. PEIERLS of the University of Oxford for useful discussions. AMS would like to thank Drs. T. W. Conlon, D. J. Findlay and J. FreEmaN of Harwell Laboratory, UK, for helpful discussions.

\section{REFERENCES}

[1] Knoll G. F., Radiation Detection and Measurement (Wiley, New York, N.Y.) 1979.

[2] Koonin S. E., Nature, 354 (1991) 468.

[3] Simpson J. J., Phys. Rev. Lett., 54 (1985) 1891.

[4] Hime A. and Simpson J. J., Phys. Rev. D, 39 (1989) 1837.

[5] Simpson J. J. and Hime A., Phys. Rev. D., 39 (1989) 1825.

[6] Hime A. and Jelley N. A., Phys. Lett., 257 (1991) 441.

[7] WARK D., Physics World, 5 (1992) 20.

[8] Bichsel H., Rev. Mod. Phys., 60 (1988) 663.

[9] Alig R. C. and Bloom S., Phys. Rev. Lett., 35 (1975) 1522.

[10] Blakemore J. S., Semiconductor Statistics (Dover, New York, N.Y.) 1962, pp. 60 and 67.

[11] Mukoyama T., Nucl. Instrum. Methods, 134 (1976) 125.

[12] DuBors D. F., Ann. Phys. (N.Y.), 7 (1959) 174; 8 (1959) 24.

[13] Planskoy B., Nucl. Instrum. Methods, 61 (1968) 285.

[14] Ohtaka K. and Tanabe Y., Rev. Mod. Phys., 62 (1990) 929.

[15] PINEs D. and NozIÈres P., The Theory of Quantum Liquids. Vol. I: Normal Fermi Liquids (W. A. Benjamin Inc., New York, N.Y.) 1966.

[16] RAETHER H., Excitations of Plasmons and Interband Transitions by Electrons, Springer Tracts Mod. Phys., Vol. 88 (Springer, Berlin-Heidelberg-New York) 1980.

[17] Landau L., J. Phys. (Moscow), 8 (1944) 201.

[18] Stoneham A. M., Theory of Defects in Solids (Clarendon Press, Oxford) 1975.

[19] Farid B., Engel G., Dalin R. and van Haeringen W., Phys. Rev. B, 44 (1991) 13349.

[20] Wu C. S. and Moszkowski S. A., Beta Decay, Interscience Monographs and Texts in Physics and Astronomy, Vol. 16 (Interscience, New York, N.Y.) 1966. 